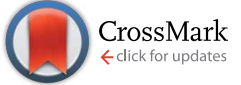

Cite this: RSC Adv., 2015, 5, 15719
Received 12th January 2015 Accepted 26th January 2015

DOI: $10.1039 / c 5 r a 00682 a$

www.rsc.org/advances

\title{
The unusual visible photothermal response of free standing multilayered films based on plasmonic bimetallic nanocages $\dagger$
}

\author{
Milana Lisunova, ${ }^{\text {a }}$ Jeremy R. Dunklin, ${ }^{\mathrm{b}}$ Samir V. Jenkins, ${ }^{\mathrm{c}}$ Jingyi Chen*c \\ and D. Keith Roper*ab
}

\begin{abstract}
An optical phenomena based on the incident light absorption and transduction to the detectable thermal signal by plasmonic bimetallic Ag and Au nanocages (Ag@AuNCs) has been researched on free standing layer-by-layer (LbL) films of poly(vinyl alcohol) (PVA) and poly(vinyl pyrrolidone) (PVPON), (PVA/ PVPON_Ag(AuNCs). Unlike well-studied monometallic Au nanocages (NCs), which possess a photothermal response in the near-infrared region, the bimetallic nanocages show a pronounced photothermal response in the visible range $(532 \mathrm{~nm})$ and near-infrared range $(780 \mathrm{~nm})$ due to the presence of two characteristic peaks at both wavelength ranges. The photothermal response in the visible range $(532 \mathrm{~nm}$ ) is distinguishable. Specifically increasing the laser power to $100 \mathrm{~mW}$ led to visual burning of the free standing film (temperature increased greater than $>150{ }^{\circ} \mathrm{C}$ ). The photothermal response by (PVA/PVPON_Ag(AAuNCs) $n$ films increases in proportion to the number $(n)$ of bilayers (bl). It also increases as the molar concentration of the Ag@AuNCs introduced to the PVPON layer is increased. Therefore the molar concentration of the plasmonic Ag(AuNCs in (PVA/PVPON_Ag@AuNCs) $n$ films is a primary factor that affects the photothermal dynamic response along with Ag@AuNCs distribution. This is supposed to result from the Ag@AuNCs assembled in a layer that leads to electromagnetic field enhancement. The unusual observation in multilayered (PVA/PVPON_Ag@AuNCs) ${ }_{n}$ films is that the UVvisible spectra (extinction efficiency) and photothermal response ( $T_{\max }$ ) do not rely on the content of the adjacent layer of PVA and show a comparable (by value in magnitude) photothermal response at a different PVA composition of $2 \mathrm{mg} \mathrm{ml}^{-1}$ and $20 \mathrm{mg} \mathrm{ml}^{-1}$ at the same Ag@AuNCs concentration in the PVPON layer.
\end{abstract}

\section{Introduction}

Metallic nanostructures with multiple surface plasmon resonance (SPR) bands across the visible and infra-red regions are of increasing interest due to their potential applications in photovoltaics, as detectors or solar cells, and in bioanalysis, as enhanced fluorescence labels, optical biosensors, or plasmonic photocatalysts. Methods to achieve multi-band plasmon resonance include tuning nanostructure geometry, ${ }^{1}$ ordering particles into lattices, ${ }^{2-5}$ combining metals with different optical properties $^{6-9}$ and composing core-shell nanostructures. ${ }^{\mathbf{1 0 , 1 1}}$ Nanorod geometries, for example, possess both longitudinal

\footnotetext{
${ }^{a}$ Ralph E. Martin Department of Chemical Engineering, 3202 Bell Engineering Center, USA. E-mail: lisunova@uark.edu; Fax: +1 479575 7926; Tel: +1 7702568426

${ }^{b}$ MicroElectronics-Photonics Program, Institute for Nanoscience and Engineering, USA. E-mail: dkroper@uark.edu; Fax: +1703 292 9051; Tel: +1703 2928769

${ }^{c}$ Chemistry and Biochemistry, University of Arkansas, Fayetteville, AR 72701, USA. E-mail: chenj@uark.edu; Fax: +1 479575 4049; Tel: +1 4795756203

$\uparrow$ Electronic supplementary information (ESI) available. See DOI: 10.1039/c5ra00682a
}

and transverse SPR modes, with the former providing the highest electromagnetic (EM) field enhancement. ${ }^{12}$ Lattice resonances supported by ordered plasmonic nanostructures can be orders of magnitude more intense than individual particles due to EM field coupling among the nanostructures in the array. ${ }^{13-16}$ Recently reported was simultaneous enhancement from bimetallic particles at different spectral ranges. ${ }^{\mathbf{1 7}}$ Specifically, the Au@Ag core-shell nanoparticles displayed multiple LSPR peaks that enhanced surface Raman scattering intensity of 1-naphthalenethiolate at three excitation wavelengths: 532, 633, and $785 \mathrm{~nm} .{ }^{17}$ However, investigation of plasmonic enhancement from bimetallic particles is in its infancy; more comprehensive studies are needed in order to utilize them for various applications.

This work has examined the photothermal response of the multi-band spectrum from plasmonic, bimetallic $\mathrm{Ag}$ and $\mathrm{Au}$ nanocages (Ag@AuNCs) that were introduced to free-standing poly(vinyl alcohol) and poly(vinyl pyrrolidone) (PVA/PVPON) layer-by-layer (LbL) films, which has been shown to be stable polyelectrolyte complexes that allow fine control over composition, thickness, and functionality. Based on the studies by 
Chen et al. the hollow plasmonic Au nanocages strongly absorb light, which results in significant photothermal response, ${ }^{\mathbf{1 8 , 1 9}}$ particularly suitable for controlled release of $\operatorname{drug}^{20}$ and photothermal cancer treatments. ${ }^{21}$ However, these reports mostly focused on the Au nanocages (AuNCs) exhibiting single-band SPR in the infra-red region for biomedical applications. ${ }^{22}$ The efficiency of light-to-heat conversion for Ag@AuNCs having multi-band spectrum in a visible region remains unknown. In this context, the Ag@AuNCs having two characteristic peaks at visible $(\sim 500 \mathrm{~nm})$ and near infra-red $(\sim 680 \mathrm{~nm})$ wavelengths separated by $200 \mathrm{~nm}$ are incorporated into the polymeric film. ${ }^{\mathbf{1 8}}$ The Ag@AuNC-incorporated free standing films are investigated by reflection UV-vis spectroscopy and photothermal analysis. This study is the first to examine the photothermal response of the free standing LbL films containing plasmonic nanoparticles.

\section{Results and discussion}

To facilitate homogeneous distribution in the film, the $\mathrm{Ag} @ A u N C s$ were dispersed in PVPON and deposited by a layerby-layer (LbL) approach ${ }^{23}$ along with PVA to produce stable hydrogen-bonded films (see Fig. 1 and S1-S3† shows the range of bilayer thicknesses). ${ }^{\mathbf{2 4 2 5}}$ PVA/PVPON LbL assemblies have been shown to be stable polyelectrolyte complexes that allow fine control over composition, thickness, and functionality. ${ }^{26,27}$ The Ag@AuNC dispersion was produced using a galvanic replacement of the $\mathrm{Ag}$ nanocubes by chloroauric acid $\left(\mathrm{HAuCl}_{4}\right)$ in water. ${ }^{28}$ Fig. 2a shows a transmission electron microscopy (TEM) image of the as-synthesized Ag@AuNCs. There is a substantial population of Ag@AuNCs with an edge length of $50 \pm 5 \mathrm{~nm}$ with a wall thickness of $8 \pm 2 \mathrm{~nm}$. The average composition of Ag@AuNCs was measured to be $46.3 \%$ of $\mathrm{Ag}$ and $53.7 \%$ of $\mathrm{Au}$ by atomic absorption spectroscopy (AAS). Individual assemblies compose of a bilayer of PVA and PVPON_Ag@AuNCs and are stacked into multilayers as (PVA/ PVP_Ag@AuNCs $)_{n}$.

Bimetallic Ag@AuNCs were incorporated into PVPON of the bilayer PVA/PVPON at different molar concentrations (i.e. $250 \mathrm{fM}$ and $1250 \mathrm{fM}$ ), as shown in Fig. 2b and c, respectively. A less than 5-fold increase in number of Ag@AuNCs in Fig. 2b and c results from increased aggregation as concentration of Ag@AuNCs increases. At $250 \mathrm{fM}$ most of the population appears to be individual Ag@AuNCs with some dimers. At $1250 \mathrm{fM}$, an increased number of dimers was observed. The distance between uncoupled Ag@AuNCs is typically on the order of micrometer range. Inserts in Fig. $2 \mathrm{~b}$ and $\mathrm{c}$ show clustered Ag@AuNCs increased in number and size at higher concentration. At $1250 \mathrm{fM}$, as many as 14 Ag@AuNCs are clustered; whereas at $240 \mathrm{fM}$, only clusters of $2 \mathrm{Ag} @$ AuNCs appear, at the same scale of imaging area. The tendency of anisotropic nanoparticles to agglomerate with predominantly side-to-side interaction was reported by Xia, Mirkin and collaborators. ${ }^{29-34}$ Agglomeration facet-to-facet and organization of close, compacted clusters into densely packed units (see Fig. 2c inset) rather than into linear chains was recently explained by strong van der Waals interactions between nanoparticles that have a large contact surface area. ${ }^{23,35}$ Clustering anisotropic plasmonic nanoparticles may cause variations in optical properties. For instance, recent studies of fluorescent polymers assembled on

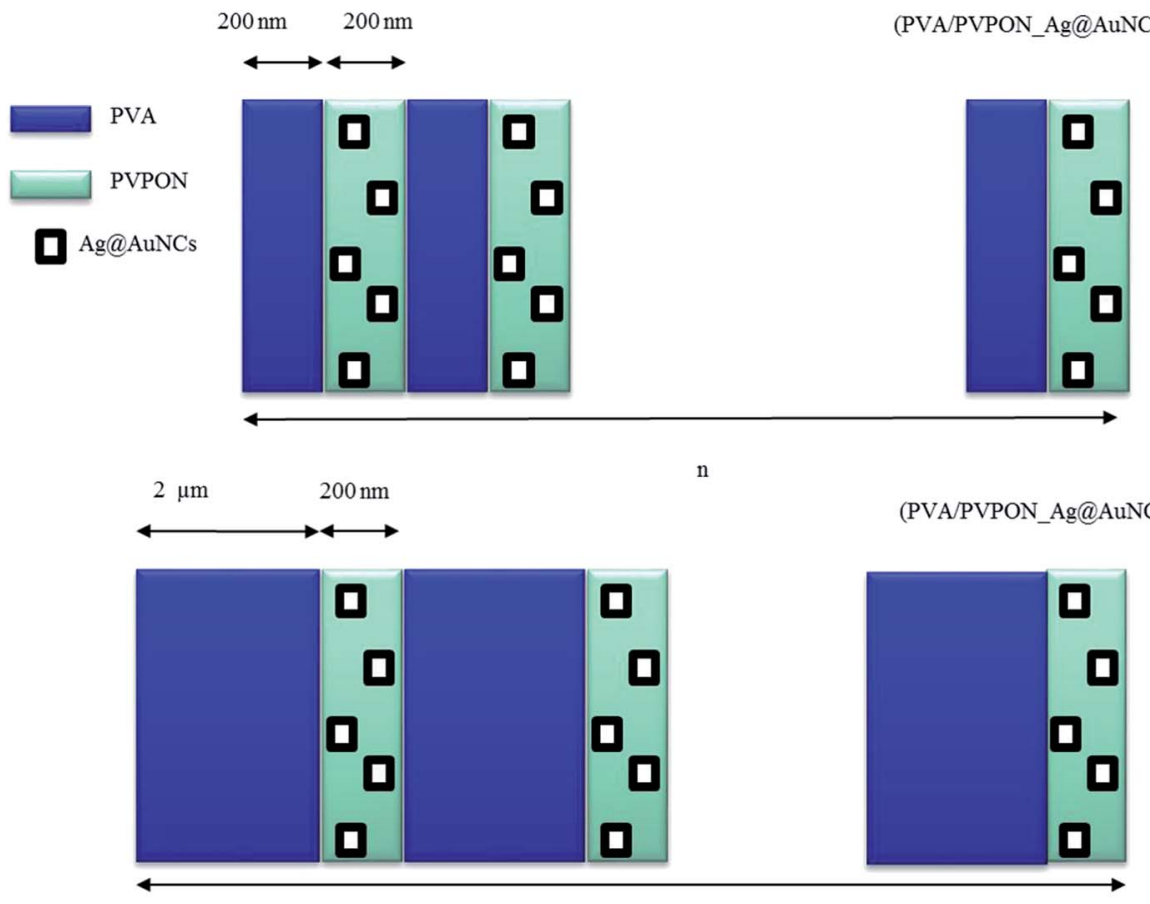

Fig. 1 Schematic illustration of the PVA/PVPON LbL free standing films with Ag@AuNCs introduced to PVPON separated by two different PVA concentrations of $2 \mathrm{mg} \mathrm{ml}^{-1}$ and $20 \mathrm{mg} \mathrm{ml}^{-1}$. 

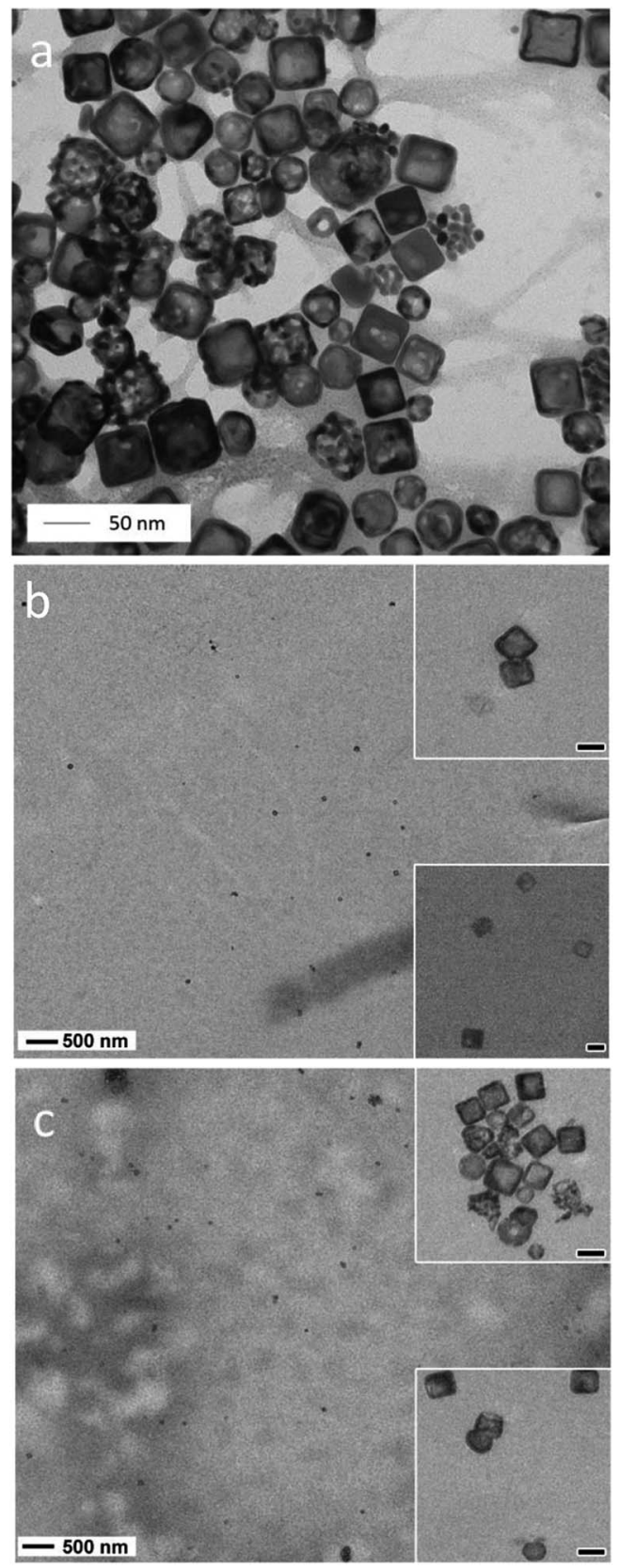

Fig. 2 TEM images of Ag@AuNCs: in the as-synthesized dispersion (a), and in PVPON layer deposited on the top of PVA with different molar concentrations of the Ag@AuNCs in bilayer PVA/PVPON of $250 \mathrm{fM}$ (b) and $1250 \mathrm{fM}$ (c). Scale bar in the inserts correspond to $50 \mathrm{~nm}$.

plasmonic core-shell dimers revealed a plasmon fluorescence enhancement due to plasmonic coupling. ${ }^{36}$ Clustering also affects melting temperature variation of the DNA assembled on the nanoparticles. ${ }^{37}$

The UV-vis spectrum of the colloidal, aqueous dispersion presents two characteristic features: a peak at $680 \mathrm{~nm}$ with a shoulder at about $512 \mathrm{~nm}$, as shown in Fig. 3a. The peak at 680 $\mathrm{nm}$ can be attributed to the part of Ag@AuNCs rich in $\mathrm{Au}$ compared to the shoulder at 512 mainly that rich in Ag. Discrete dipole approximation (DDA) in Fig. 3b show peaks near these wavelengths ${ }^{18}$ at $521 \mathrm{~nm}$ and $668 \mathrm{~nm}$ for $50 \% / 50 \%$ bimetallic
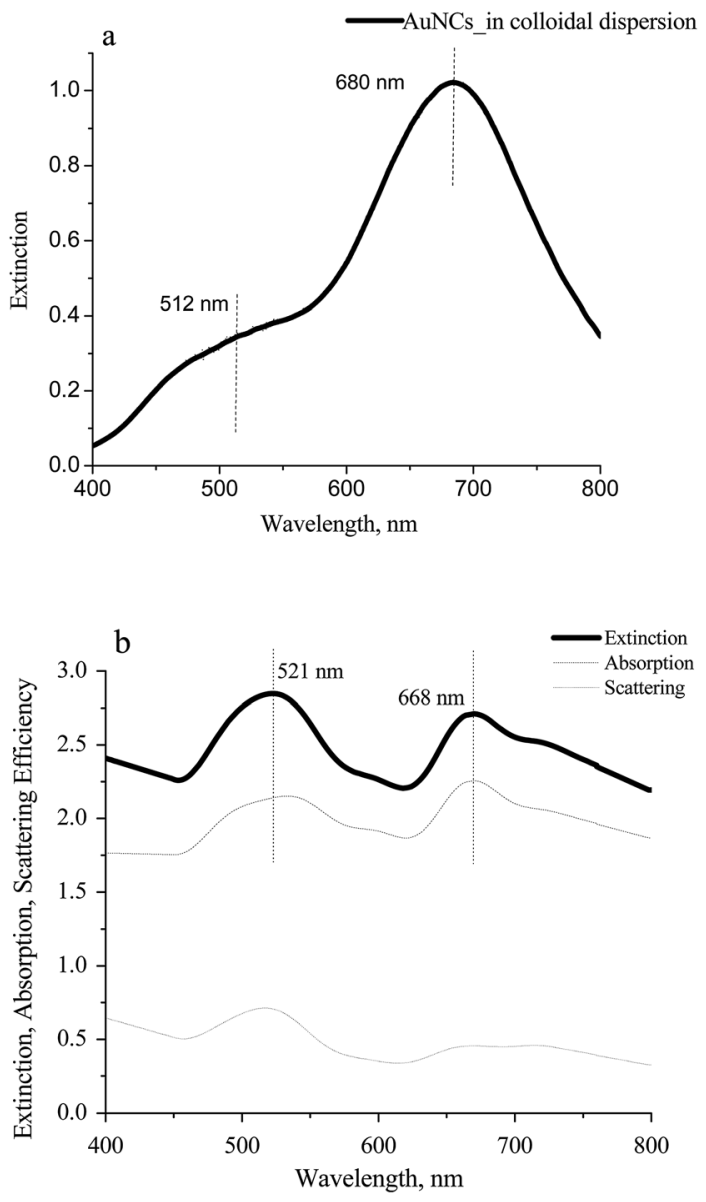

Fig. 3 Normalized UV-vis spectra of the AgaAuNCs in aqueous dispersion of $250 \mathrm{fM}$ (a). The DDA simulation of extinction, absorption and scattering efficiency spectra of hollow bimetallic Ag@Au nanocage (b).

Ag@AuNCs. This result is supported by our previous analysis of the AuNCs with a characteristic wavelength of $668 \mathrm{~nm}^{38} \mathrm{An}$ absorption/scattering ratio is about 4 for both 521 and $668 \mathrm{~nm}$ peaks. This is consistent with the fact that hollow nanoparticles primarily exhibit absorptive behavior under resonant illumination. ${ }^{39}$ The shape and aspect ratio determine the absorption and scattering. Nanoparticles shaped as hollow nanocages and a solid rod, despite similar effective volume, possess different scattering/absorption ratios. ${ }^{40}$ Compared to simulated spectra, the nonequivalent intensities and broadened bandwidths in measured spectrum could arise from the size and shape distribution of Ag@AuNCs.

Introduction of bimetallic Ag@AuNCs to multilayered polymer films appears to redshift the peak that corresponds to the Au-rich component in Ag@AuNCs about $60 \mathrm{~nm}$ from $680 \mathrm{~nm}$ in suspension to $740 \mathrm{~nm}$ in LbL film (see Fig. 4). There is an $8 \mathrm{~nm}$ redshift in the Ag-rich component peak from $512 \mathrm{~nm}$ to $520 \mathrm{~nm}$. Shifts in plasmonic peak wavelengths due to their assembly in polymer layer are confounded by significant broadening of each peak. The broadening becomes more significant with the increasing concentration of the nanoparticles in a PVPON from $250 \mathrm{fM}$ (see Fig. 4a) to $2500 \mathrm{fM}$ (see Fig. 4c). It also significantly 

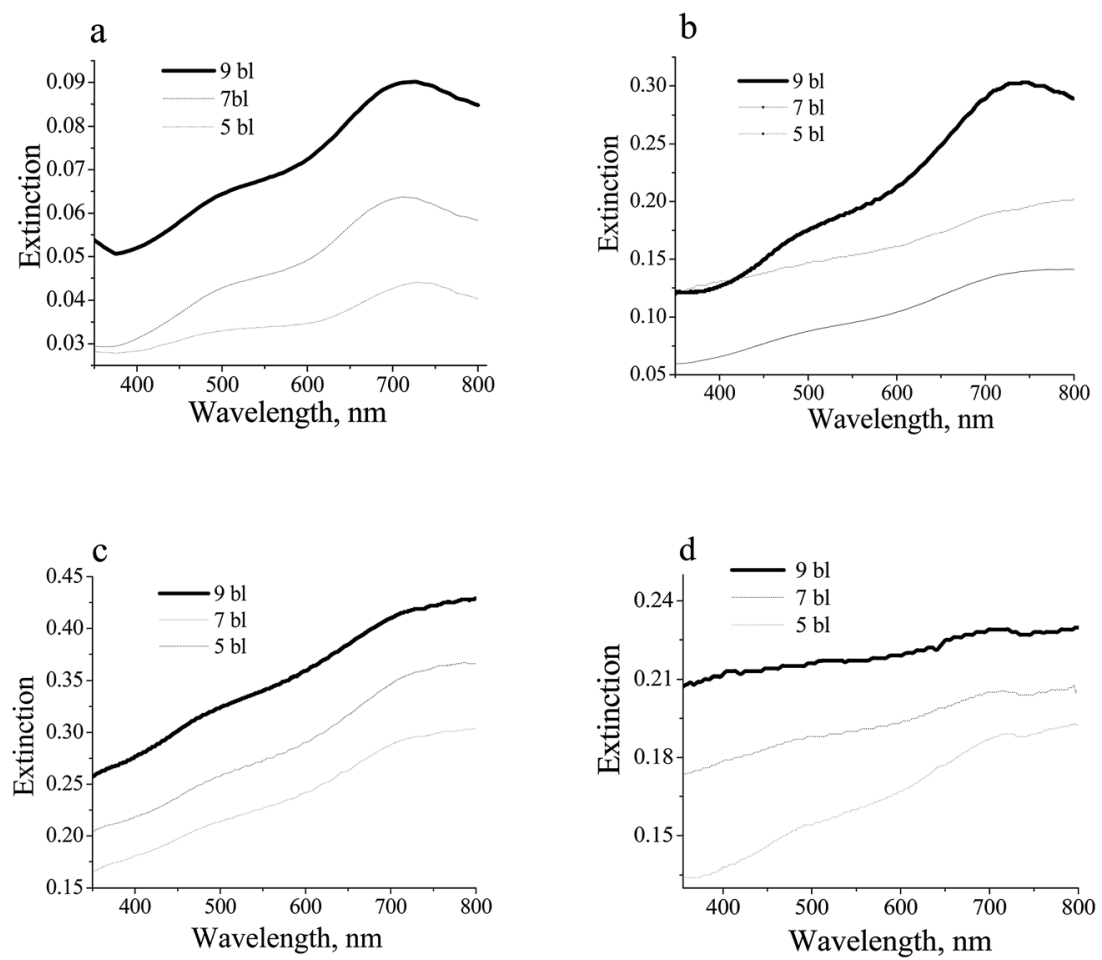

Fig. 4 UV-vis spectra of the (PVA/PVPON_Ag(auNCs) $n=5,7$ and 9 bilayers (bl) obtained from $2 \mathrm{mg} \mathrm{ml}^{-1}$ of PVPON and $2 \mathrm{mg} \mathrm{ml}^{-1}$ of PVA with molar concentrations of Ag@AuNCs in a bilayer of: $250 \mathrm{fM}$ (a); $1250 \mathrm{fM}$ (b); $2500 \mathrm{fM}$ (c). UV-vis spectra of the (PVA/PVPON_Ag@AuNCs) $n=5,7$ and $9 \mathrm{bl}$ produced on $20 \mathrm{mg} \mathrm{ml}^{-1}$ of PVA and $2 \mathrm{mg} \mathrm{ml}^{-1}$ of PVPON with a molar concentration of Ag(AuNCs in a single bilayer of $1250 \mathrm{fM}$ (d).

increases with improving PVA content from $2 \mathrm{mg} \mathrm{ml}^{-1}$ (see Fig. 4b) to $20 \mathrm{mg} \mathrm{ml}^{-1}$ (see Fig. 4d) at the same concentration as the Ag@AuNCs in a PVPON of $1250 \mathrm{fM}$. The broadening could be attributed to the inhomogeneity between the polymer layers (such as air between two phases) and polymer layer itself which leads to refractive index variation of the surrounding medium with respect to plasmonic Ag@AuNCs. The inhomogeneity increases with increasing content of the PVA (polymer layer) which leads to further broadening of the UV-vis spectra.

Overall, however, the peak shifts appear consistent with relevant reports. For example, Mahmoud et al. ${ }^{41}$ showed that increasing coverage area of a monolayer of uniform AuNCs in a monolayer from $4 \%$ to $29 \%$ redshifted the plasmonic peak from $675 \mathrm{~nm}$ to $691 \mathrm{~nm}$. In addition, assembling the polymer on AuNCs ( $4 \%$ of the AuNCs in a monolayer), redshifted the plasmonic spectra from 675 to $693 \mathrm{~nm} .{ }^{41}$ Redshifting of the SPR spectrum of the AuNCs as the percent of AuNCs coverage increased was explained by the growth of the interparticle SPR field coupling as interparticle distance decreased. The assembling of the polymer multilayers on $\mathrm{Ag}$ cubes $^{36}$ led to the appearance of a second peak attributed to particle agglomeration (dimer formation). The redshift in UV-vis spectra of (PVA/ PVPON_Ag@AuNCs) $)_{n}$, where $n$ is a number of the bilayers, may result from Ag@AuNCs aggregation as well as from increasing refractive index from 1.33 (water) to 1.49-1.51 (polymer (PVA, PVPON)). ${ }^{42,43}$ According to Tsukruk, the degree of SPR peak shift increased with the aggregation state of $\mathrm{Ag}$ nanocubes. ${ }^{42} \mathrm{~A}$ monomer extinction peak redshifted $3.1 \mathrm{~nm}$ due to a polymer shell coating of $55 \mathrm{~nm}$ while the peak assigned to an agglomerate redshifted $23.7 \mathrm{~nm}$. According to Mahmoud et al., the observed unsymmetrical red-shift behavior of the two extinction peaks may be explained by the presence of two plasmon fields (inside and outside of AuNCs). ${ }^{41}$ One study reported redshifts in multiband plasmonic spectra due to switching surrounding medium (with refractive index) from aqueous dispersion to dry film on ITO substrate. ${ }^{44}$ Liz-Marzan group showed simultaneous shift of both peaks from bimetallic Au@Ag nanorods in contrast to Ag@AuNCs which showed a pronounced redshift of only one extinction peak. This could be explained by different coupling mechanisms between Au and $\mathrm{Ag}$ in hollow cages and nanorods. ${ }^{44}$

Hollow plasmonic nanoparticles are particularly sensitive to local variations in a refractive index. For instance, Au nanoframes possess a sensitivity factor of about $620 \mathrm{~nm}$ per RIU which is one order of magnitude higher compared to $\mathrm{Au}$ nanocubes with a sensitivity factor of about 40 (nm per RIU). ${ }^{45}$ Our bimetallic Ag@AuNCs shows a chemical sensitivity of about $333 \mathrm{~nm}$ per RIU. Specifically, the refractive index difference between water medium and polymer multilayers is $0.18 \mathrm{RIU}$ and the plasmon shift is $60 \mathrm{~nm} .60 \mathrm{~nm}$ divided by 0.18 RIU equals $333 \mathrm{~nm}$ per RIU, assuming that the redshift was caused only by a variation of the refractive index. The increased sensitivity can be explained by plasmon field coupling between their interior and exterior surface fields. ${ }^{45,46}$ Anisotropic shapes of these nanoparticles can increase the sensitivity to the refractive index of the surrounding medium. For example, Au nanostars have sharp edges that enhance the adjacent EM field and have a sensitivity of $670 \mathrm{~nm}$ per RIU. ${ }^{47}$ The high sensitivity of plasmon 
peak variations with respect to the refractive index change of the medium may be potentially important in manipulating photothermal sensitivity in a broad optical range.

It is also important to note that the extinction intensity in a layered structure increases with the increasing amount, $n$, of the multilayers (PVA/PVPON_Ag@AuNCs) ${ }_{n}$ (see Fig. 4). Extinction intensity in layered structure also increases as molar concentration of the nanoparticles increases in PVPON layer (see Fig. 4a-c). Such observations are consistent with those reported previously for polymer composite of plasmonic nanoparticles. ${ }^{50,53,57,58}$ However, in high contrast to the composites (homogeneous mixture of the polymer and nanoparticles), the extinction intensity in a layered structure (see Fig. 1) does not rely on the content of adjacent polymer layer (see Fig. $4 \mathrm{~b}$ and d). This observation is of particular interest for further research of the incoming light absorption by layered film and its transduction to the thermal energy.

The photothermal response is evaluated as the function of four factors: (1) molar concentration of the Ag@AuNCs (see Table 1); (2) content of the adjacent PVA layer (see Fig. 1 and S1S3 $\uparrow$ show the range of bilayer thicknesses); (3) the method of introducing Ag@AuNCs to the free standing film; and (4) laser wavelengths of $532 \mathrm{~nm}$ and $780 \mathrm{~nm}$ near the spectral extinction maxima of about $520 \mathrm{~nm}$ and 720 to $750 \mathrm{~nm}$. Overall photon-toheat conversion provides a measure of dissipated heat from excited nanoparticles to the polymer free standing film. ${ }^{48-50}$ Direct evaluation of the Ag@AuNCs temperature variation, due to plasmonic excitation, is precluded by an ultra-fast heat dissipation (100-380 ps) ${ }^{51}$ from the highly thermoconductive Ag@AuNCs (ca. 100-60 $\left.\mathrm{W} \mathrm{m}^{-1} \mathrm{~K}^{-1}\right)$ as well as ultra-low time relaxation of about $<10^{-9} \mathrm{S.}^{49,52-54}$

Measurements conducted with green and red lasers at wavelengths of $532 \mathrm{~nm}$ and $780 \mathrm{~nm}$, respectively, demonstrated that the (PVA/PVPON_Ag@AuNCs) ${ }_{n}$ are active for both laser radiations. Such multi-photothermal responsiveness could be the result of there being similar absorption to scattering yield for the Ag@AuNCs at both extinction peaks (see Fig. 3b) and highly scattering, aggregated Ag@AuNCs at infrared wavelengths. Based on the Roper group experience in consistent data generation by a facility with a green laser the subsequent consideration is based on the green laser (532 nm). Applied power for the laser was about $25 \mathrm{~mW}$ with the laser spot of about $1 \mathrm{~mm}$ in diameter; subsequently, increasing laser power to $100 \mathrm{~mW}$ led visual to a burning of the samples. This indicated a temperature increase greater than $>150{ }^{\circ} \mathrm{C}$, because of the melting point of PVPON $\left(T_{\mathrm{m}}=150^{\circ} \mathrm{C}\right)$ and PVA $\left(T_{\mathrm{m}}=230^{\circ} \mathrm{C}\right)$. Phase transformation due to plasmonic heating has been reported previously. Plasmonic, optothermal water evaporation $^{24,55,56}$ and other optofluidic effects have also been reported. ${ }^{55,57,58}$ Soft, biocompatible polymers have been reported by Roper et al. to maximize thermoplasmonic transduction in proportion to area density of plasmonic nanostructures. ${ }^{50,59}$ The resonant thermalization by nanoparticles could treat cancer cells as well. ${ }^{60-64}$

Fig. 5 shows the photothermal response of (PVA/ PVPON_Ag@AuNCs $)_{n}$ with different amount $(n)$ of the bilayers (bl) of $5 \mathrm{bl}, 7 \mathrm{bl}$, and $9 \mathrm{bl}$ and same the molar concentration in a single layer $1250 \mathrm{fM}$ versus PVA content of $2 \mathrm{mg} \mathrm{ml}^{-1}$ and $20 \mathrm{mg} \mathrm{ml}^{-1}$. The photothermal response (Fig. 5a) increases in proportion with an increasing number of bilayers for both composites with different PVA content of $2 \mathrm{mg} \mathrm{ml}^{-1}$ and $20 \mathrm{mg} \mathrm{ml}{ }^{-1}$, from $28{ }^{\circ} \mathrm{C}$ to $36.6{ }^{\circ} \mathrm{C}$ and from $28{ }^{\circ} \mathrm{C}$ to $35.5^{\circ} \mathrm{C}$, respectively. These correspond to increases of 344 and $300{ }^{\circ} \mathrm{C}$ per Watt, respectively. The content of PVA does not affect temperature response which is in high contrast to a composite based on the mixture of the components. Specifically, the increasing amount of the insulator in the composite leads to a reduction of the plasmonic filler; therefore, reducing the light absorption efficiency and its transduction to the thermal energy. ${ }^{50,59}$ In the case of the multilayered structure the absorption efficiency does not change significantly with content of PVA (see Fig. 4b and d); therefore, the observed photothermal responses $\left(T_{\max }\right)$ are comparable. However, the time response (time at which system reaches the maximum value) escalation from $0.5 \pm 0.1 \mathrm{~s}$ to $>1.6 \pm 0.2 \mathrm{~s}$ with amount increasing PVA layer concentration is understandable from thermal mass enhancement (Fig. 5b). Increasing the molar concentration of $\mathrm{Ag} @$ AuNCs introduced to each PVPON layer increases the

Table 1 Molar concentration of Ag@AuNCs introduced to PVPON of free standing (PVA/PVPON_Ag@AuNCs) films (2 cm by $1 \mathrm{~cm}$ in size), temperature and time response

\begin{tabular}{|c|c|c|c|c|}
\hline Number bilayers & $\begin{array}{l}\text { Molar concentration } \\
\text { in single PVPON layer }(\mathrm{fM}) \\
\left(10^{-12} \mathrm{~mol} \mathrm{~m}^{-3}\right)\end{array}$ & $\begin{array}{l}\text { Total molar } \\
\text { concentration in film (pM) } \\
\left(10^{-9} \mathrm{~mol} \mathrm{~m}^{-3}\right)\end{array}$ & $T_{\max }\left({ }^{\circ} \mathrm{C}\right)$ & Time response $(\mathrm{s})$ \\
\hline \multirow[t]{3}{*}{$5 \mathrm{bl} 2 \mathrm{mg} \mathrm{ml}^{-1}$} & 2500 & 12.5 & 31 & 0.3 \\
\hline & 1250 & 6.25 & 28 & 0.4 \\
\hline & 250 & 1.25 & $\mathrm{~N} / \mathrm{A}$ & 1.4 \\
\hline \multirow{4}{*}{$\begin{array}{l}5 \mathrm{bl} 20 \mathrm{mg} \mathrm{ml}^{-1} \\
7 \mathrm{bl} 2 \mathrm{mg} \mathrm{ml}^{-1}\end{array}$} & 1250 & 6.25 & 28 & 1.4 \\
\hline & 2500 & 17.5 & 33 & 0.7 \\
\hline & 1250 & 8.75 & 30 & 0.7 \\
\hline & 250 & 1.75 & 24.1 & \\
\hline \multirow{4}{*}{$\begin{array}{l}\text { 7bl } 20 \mathrm{mg} \mathrm{ml}^{-1} \\
9 \mathrm{bl} 2 \mathrm{mg} \mathrm{ml}^{-1}\end{array}$} & 1250 & 8.75 & 30 & 1.6 \\
\hline & 2500 & 22.5 & 37.6 & 0.5 \\
\hline & 1250 & 11.25 & 36.6 & 0.7 \\
\hline & 250 & 2.25 & 25 & 0.7 \\
\hline $9 \mathrm{bl} 20 \mathrm{mg} \mathrm{ml}^{-1}$ & 1250 & 11.25 & 35.5 & 1.8 \\
\hline
\end{tabular}



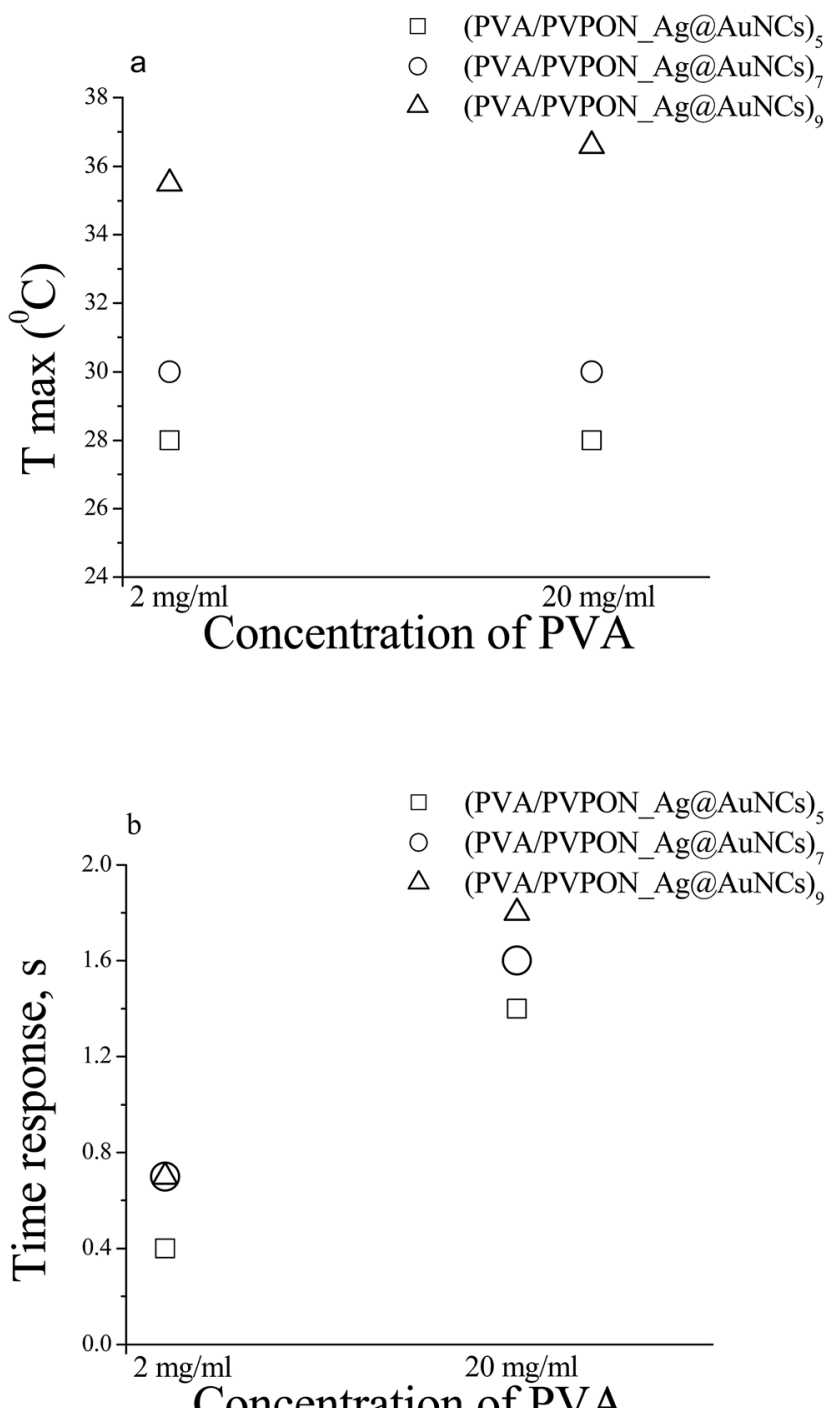

Fig. 5 Static photothermal response of (PVA/PVPON_Ag(AuNCs) versus the PVA layer content at $2 \mathrm{mg} \mathrm{ml}^{-1}$ vs. $20 \mathrm{mg} \mathrm{ml}^{-1}$ at same molar concentration of Ag@AuNCs in a single bilayer of $1250 \mathrm{fM}$ (a). Dynamic time response of (PVA/PVPON_Ag(AuNCs) $n$ versus the PVA layer content at $2 \mathrm{mg} \mathrm{ml}^{-1} \mathrm{vs} .20 \mathrm{mg} \mathrm{ml}^{-1}$ at same molar concentration of AgaAuNCs in a single bilayer of $1250 \mathrm{fM}$ (b).

temperature response (see Fig. 6). Specifically, the maximum temperature attained increased from $25.0^{\circ} \mathrm{C}$ to $37.6{ }^{\circ} \mathrm{C}$ for $9 \mathrm{bl}$ films and from $25.0{ }^{\circ} \mathrm{C}$ to $36.0{ }^{\circ} \mathrm{C}$ for $7 \mathrm{bl}$ films as the molar concentration of $\mathrm{Ag} @ A$ ANCs in each layer increased from 250 $\mathrm{fM}$ to $2500 \mathrm{fM}$. In each case the ambient temperature was 21.0 ${ }^{\circ} \mathrm{C}$ and the applied laser power was about $25 \mathrm{~mW}$.

Based on recent reports by El-Sayed and collaborators, interparticle separation contributes to plasmon field coupling, ${ }^{\mathbf{4 1 , 4 2}}$ which consequently may affect the optical properties of the hybrid nanostructures. In order to assess the effects of the coupling on plasmon field enhancement the Ag@AuNCs were simulated in a regime of close proximity (of about $2 \mathrm{~nm})^{65}$ and at a large separation (of about $200 \mathrm{~nm}) .{ }^{41}$ Fig. 7 shows plasmon field contours of dimers $2 \mathrm{~nm}$, and $>200 \mathrm{~nm}$ apart. These EM field distributions were induced by optical excitation

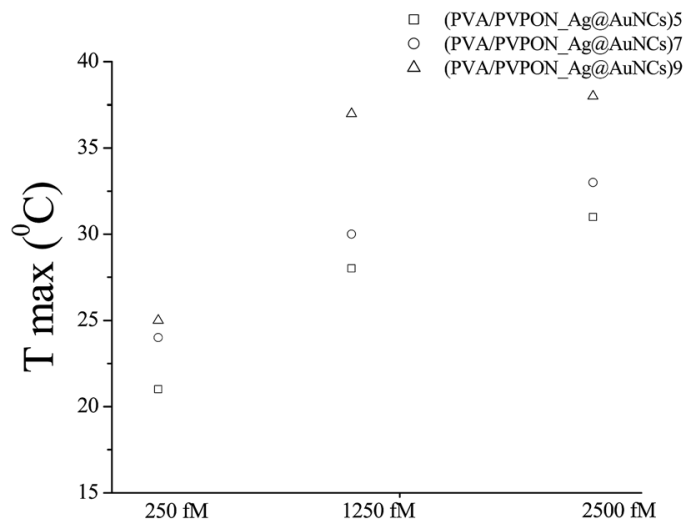

Molar concentration of Ag@AuNCs in single PVPON layer

Fig. 6 Static photothermal response of (PVA/PVPON_Ag@AuNCs) (PVA/PVPON_Ag@AuNCs) ${ }_{7}$ and (PVA/PVPON_Ag(AuNCs) ${ }_{9}$ versus the molar concentration of Ag(AuNCs introduced to a bilayer.

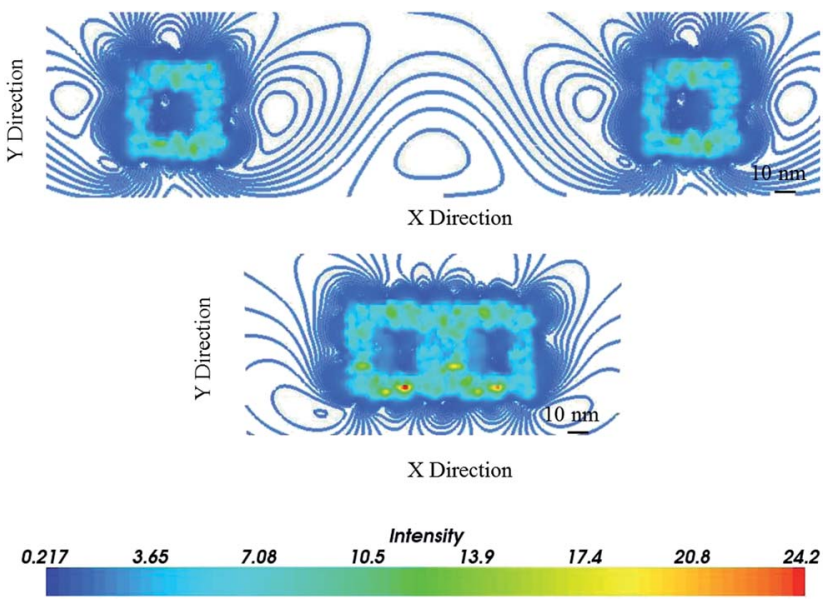

Fig. 7 The plasmon field contour of the dimers bimetallic Ag@AuNCs excited at $521 \mathrm{~nm}$ at a large interparticle distance of (top) $200 \mathrm{~nm}$ and (bottom) in a regime of the close proximity of $2 \mathrm{~nm}$.

of SPR of Ag@AuNCs. The simulations show the plasmonic field is enhanced at the wall with an additional increase between the particles at $2 \mathrm{~nm}$ that decreases with an increasing interparticle separation. The Ag@AuNC dimers at $200 \mathrm{~nm}$ apart have no observable EM field enhancement between the nanocages; the EM field distribution is identical on the walls and corners of both nanocages. This is consistent with the results by Mahmoud et al. ${ }^{41}$ for AuNCs separated by $180 \mathrm{~nm}$ (no interminable EM field enhancement) and $60 \mathrm{~nm}$ (enhanced interminable EM field).

The result is also consistent with previous reports in AuNCs by Chen et al. ${ }^{66}$ Specifically, the photothermal response of the colloidal dispersion of the nanocages increased from $\Delta T=$ $10{ }^{\circ} \mathrm{C}$ to $45{ }^{\circ} \mathrm{C}$ with its concentration increase from 1 to 5 , and $150 \mathrm{pM}$ at incident laser power of $1 \mathrm{~W} \mathrm{~cm}^{-2}$. The higher value of $\Delta T$ in magnitude in aqueous dispersion in contrast to solid layered film could be explained by higher molar concentration (in one order of magnitude) of the introduced Ag@AuNCs (see 
Table 1). We also should notice that method of the photothermal response evaluation also affect the measured values.

It is worth nothing that the photothermal response of the multilayered film (PVA/PVPON_Ag@AuNCs) g $_{9}$ of about $700{ }^{\circ} \mathrm{C} \mathrm{W}^{-1}$ is twice less of the recently reported Au continuous monolayer produced by electroless plating of about $1400{ }^{\circ} \mathrm{C} \mathrm{W}^{-1} .^{48}$ The highest thermal response reported to date of $3000{ }^{\circ} \mathrm{C} \mathrm{W}^{-1}$ by Dunklin et al. ${ }^{59}$ appeared to be due to an increase in area density of reduced gold into a $7 \mu \mathrm{m}$ skin on a $130 \mu \mathrm{m}$ thick polydimethylsiloxane (PDMS) film. The latter asymmetric Au-PDMS films exhibit 3-, 11-, and 230-fold larger thermal response in comparison with reduced TCA, annealed $\mathrm{Au}$ films, or suspended $\mathrm{Au}$ nanoparticles, respectively, which have uniformly distributed particles. It is well known that defects and dislocations in solid bodies may lead to reducted efficiency of the thermal energy transduction. ${ }^{67-69}$ In order to get a superior photothermal response, a future study should be performed with focus on photothermal responses in a continuous monolayer ${ }^{70}$ with homogeneous distribution of nanocages along with inter-nanocages coupling.

In conclusion, in high contrast to previous reports which focused on photothermal effects at infrared wavelengths by AuNCs, the research in bimetallic Ag@AuNCs shows an additional distinguishable photothermal response at visible wavelength range. Such an effect is observed in bimetallic $\mathrm{Ag} @ A u N C s$ explained by the existence of two extinction peaks at visible and near infra-red wavelength range with similar absorption to scattering yield. The photothermal response by the free standing film (PVA/PVPON_Ag@AuNCs) $)_{n}$ increased as the molar concentration of the $\mathrm{Ag} @ A$ AuNCs introduced to PVPON layer increased (from $25{ }^{\circ} \mathrm{C}$ to $38{ }^{\circ} \mathrm{C}$ with the concentration increasing from $250 \mathrm{fM}$ to $2500 \mathrm{fM}$ in the PVPON layer of (PVA/PVPON_Ag@AuNCs) $)_{9}$ ). The concentration of the plasmonic Ag@AuNCs in multilayered free standing films was found to be a key factor that affects the photothermal response along with its distribution, because of the addition EM field coupling in clusters. The increasing laser power to $100 \mathrm{~mW}$ led to visual burning of the free standing film (temperature increase greater than $>150{ }^{\circ} \mathrm{C}$ ). The photothermal response of the multilayered (PVA/PVPON_Ag@AuNCs) ${ }_{n}$ films does not rely on the content of the adjacent PVA layer which is unusual to our previous observation in photothermal responses of the homogeneous composites. The photothermal time response, at which the film reaches the maximum value, increases from $0.5 \pm 0.1 \mathrm{~s}$ to $1.6 \pm 0.2 \mathrm{~s}$ with increasing adjacent PVA layer content from $2 \mathrm{mg} \mathrm{ml}^{-1}$ and $20 \mathrm{mg} \mathrm{ml}^{-1}$ for all bilayer $(n=5 \mathrm{bl}, 7 \mathrm{bl}$ and $9 \mathrm{bl})$.

\section{Experimental section}

\section{Ag@AuNCs synthesis}

Bimetallic silver gold nanocages (Ag@AuNCs) were synthesized by using $\mathrm{Ag}$ cubes as a sacrificial template for the galvanic replacement reaction. Specifically, Ag nanocubes were synthesized according to the well known polyol method. As synthesized Ag nanocubes ( $96 \mathrm{pmol}, \sim 8 \mathrm{nM}$ ) with edge length of $\sim 40 \mathrm{~nm}$ and local surface plasmon resonance peak (LSPR) at $443 \mathrm{~nm}$ (ref. 71) were titrated with $1 \mathrm{mM} \mathrm{HAuCl}_{4}$ aqueous solution at a rate of $45 \mathrm{ml} \mathrm{h}^{-1}$ at $100{ }^{\circ} \mathrm{C}$ under vigorous stirring until the LSPR of the reaction mixture reached $\sim 673 \mathrm{~nm} .{ }^{72}$ After cooling to room temperature the AuNCs were further purified with saturated $\mathrm{NaCl}$ solution to remove $\mathrm{AgCl}$ precipitate. The product was washed twice with $\mathrm{H}_{2} \mathrm{O}$ and redispersed in $\mathrm{H}_{2} \mathrm{O}$ for future use. Based on the atomic absorption data the AuNCs contain $46.3 \%$ of $\mathrm{Ag}$ and $53.7 \%$ of $\mathrm{Au}$.

\section{Samples preparation}

PVA (molecular weight $(\mathrm{MW})=30 \mathrm{kDa})$, PVPON of molecular weight $(40 \mathrm{kDa})$ were purchased from Sigma-Aldrich. The layerby-layer deposition of (PVA/PVPON_Ag@AuNCs) $)_{n}$ multilayers has been performed according to the established procedure described elsewhere (Fig. 1). ${ }^{73}$ Briefly, $20 \mathrm{mg} \mathrm{ml} \mathrm{ml}^{-1}$ and $2 \mathrm{mg} \mathrm{ml}^{-1}$ PVA solution were prepared by dissolving polymer in ultrapure water. Ag@AuNCs were dispersed in $2 \mathrm{mg} \mathrm{ml}$ PVPON solution in ultrapure water at concentration of $250 \mathrm{fM}$, $1250 \mathrm{fM}$ and $2500 \mathrm{fM}$. Typical deposition time was $4 \mathrm{~h}$. The rinsing steps had been precluded in order to keep stable concentration of nanoparticles and thickness of the bilayers. The rinsing may cause detachment of the adhered nanoparticles. Moreover a recent publication by Suntivich et al. proves a possibility to construct a homogeneous multilayered LbL films without an intermediate washing step. ${ }^{74}$

\section{Spectroscopy and imaging}

UV-visible spectra of the colloidal dispersions were recorded using a UV-vis HP 8453 spectrophotometer (Agilent). UV-visible spectra of the specimen were recorded using a UV-vis 1800 spectrophotometer (Shimadzu). The concentrations of $\mathrm{Au}$ and Ag were determined using a GBC 932 atomic absorption (AA) spectrometer. Transmission electron microscopy (TEM) imaging of $\mathrm{Au}$ nanocages and coated with polymers was performed on a JEOL 100cx operated at $100 \mathrm{kV}$. Before imaging, Au nanocages coated with polymers were drop cast on the carbonformvar TEM grid (Electron Microscopy Sciences) and dried in air. SEM was performed using a Philips XL40 FEG SEM.

\section{Photothermal properties measurement}

Temperature variation was captured using $1 \mathrm{~cm} \times 2 \mathrm{~cm}$ samples of the films suspended horizontally with the laser spot $(1 \mathrm{~mm}$ diameter) centered within the film. The camera that captures temperature variation (ICI 7320, Infrared Cameras Inc., Beaumont, TX, USA) was placed behind the sample, opposite the laser. The utilized approach is similar a previously reported. ${ }^{48,50,59}$ The two lasers with wavelength of $532 \mathrm{~nm}$ (MXLFN532, CNI, Changchung, CN) and $780 \mathrm{~nm}$ (100 mW Infrared Laser Diode Module Lazer Dot, Laserlands, China) have been used. However due to the instability of the infrared laser a more systematical data have been conducted with green laser witch is stable. Laser power was monitored before and after each trial to make sure it remained constant. The laser power was set to $25 \mathrm{~mW}$ to keep the infrared camera within its operational temperature range $\left(<100{ }^{\circ} \mathrm{C}\right)$. The infrared camera recorded the thermal data at $10 \mathrm{~Hz}$ within 1-120 s heating period (depending 
on the sample composition) with the laser on and a 1-120 s cool down period with the laser turned off.

\section{Simulations}

The discrete dipole approximation (DDA) package DDSCAT method was utilized for simulation. ${ }^{38,75}$ The calculations here refer to water as the external dielectric medium. The dispersive gold $(\mathrm{Au})$ and silver $(\mathrm{Ag})$ dielectric values in all calculations were taken from Johnson and Christy. ${ }^{76}$

The shape files of hollow cube ${ }^{\mathbf{1 8}}$ had been simulated via utilization Matlab. The plasmonic spectra of the hollow cube were evaluated via the reading shape file by DDSCAT, specifically by FROM_FILE (geometry read from shape file).

\section{Acknowledgements}

This work was supported in part by NSF ECCS-1006927, NSF CBET 1134222 and the University of Arkansas Foundation. UVvisible spectroscopy was supported by NSF EEC-1138248. The Arkansas Bio Nano Materials Characterization Facility is supported in part by the NSF. We thank Justin Norman and Drew F DeJarnette for providing comments to DDA calculations.

\section{References}

1 Z. Guo, X. Fan, L. Liu, Z. Bian, C. Gu, Y. Zhang, N. Gu, D. Yang and J. Zhang, J. Colloid Interface Sci., 2010, 348, 29.

2 M. Lisunova, J. Norman, P. Blake, G. T. Forcherio, D. F. DeJarnette and D. K. Roper, J. Phys. D: Appl. Phys, 2014, 46, 241.

3 P. Blake, J. Obermann, B. Harbin and D. K. Roper, IEEE Sens. J., 2011, 11, 3332.

4 P. Blake, S. Kuhne, G. T. Forcherio and D. K. Roper, J. Nanophotonics, 2014, 8, 083084.

5 D. DeJarnette, P. Blake, G. T. Forcherio and D. K. Roper, J. Appl. Phys., 2014, 115, 024306.

6 V. S. K. Chakravadhanula, M. Elbahri, U. Schurmann, H. Takele, H. Greve, V. Zaporojthenko and F. Faupel, Nanotechnology, 2008, 19, 225302.

7 J. Zhu, F. Zhang, J.-J. Li and J. W. Zhao, Gold Bull., 2014, 47, 47.

8 J. Zhu, J.-J. Li and J.-W. Zhao, Plasmonics, 2013, 8, 1493.

9 J. Zhu, J.-J. Li, L. Yuan and J.-W. Zhao, J. Phys. Chem. C, 2012, 116, 11734.

10 M. Lisunova, M. Mahmoud, N. Holland, Z. Combs, M. A. ElSayed and V. V. Tsukruk, J. Mater. Chem., 2012, 22, 16659.

11 C. Ayala-Orozco, C. Urban, M. W. Knight, A. S. Urban, O. Neumann, S. W. Bishnoi, S. Mukherjee, A. M. Goodman, H. Charron, T. Mitchell, M. Shea, R. Roy, S. Nanda, R. Schiff, N. J. Halas and A. Joshi, ACS Nano, 2014, 8, 6372.

12 G. Hoi Gu, M. Y. Kim, H. J. Yoon and J. S. Suh, Bull. Korean Chem. Soc., 2014, 35, 725.

13 S. Zou and G. C. Schatz, Chem. Phys. Lett., 2005, 403, 62.

14 D. K. Roper, W. Ahn, B. Taylor and Y. D'Asen, IEEE Sens. J., $2010,10,531$.
15 D. DeJarnette, D. K. Roper and B. Harbin, J. Opt. Soc. Am. B, 2012, 29, 88.

16 D. DeJarnette, J. Norman and D. K. Roper, Appl. Phys. Lett., 2012, 101, 193104.

17 A. K. Samal, L. Polavarapu, S. Rodal-Cedeira, L. M. LizMarzan, J. Perez-Juste and I. Pastoriza-Santos, Langmuir, 2013, 29, 15076.

18 M. Lisunova, J. Norman, X. Wei, S. Jenkins, J. Chen and D. K. Roper, Mater. Lett., 2014, 117, 241.

19 M. Hu, J. Chen, Z.-Y. Li, L. Au, G. V. Hartland, X. Li, M. Marqueze and Y. Xia, Chem. Soc. Rev., 2006, 35, 1084.

20 S. Y. Mustafa, et al., Nat. Mater., 2009, 8, 935.

21 J. Chen, C. Glaus, R. Laforest, Q. Zhang, M. Yang, M. Gidding, M. J. Welch and Y. Xia, Small, 2010, 6, 811.

22 M. Hu, J. Chen, Z.-Y. Li, L. Au, G. V. Hartland, X. Li, M. Marqueze and Y. Xia, Chem. Soc. Rev., 2006, 35, 1084.

23 M. Lisunova, A. Dorokhin, N. Holland, V. V. Shevchenko and V. V. Tsukruk, Soft Matter, 2013, 9, 3651.

24 Multilayer Thin Films, ed. G. Decher and J. Schlenoff, WileyVCH, Weinheim, 2012.

25 C. Jiang, S. Markutsya and V. V. Tsukruk, Langmuir, 2004, 20, 882.

26 S. Yang, Y. Zhang, X. Zhang and J. Xu, Soft Matter, 2007, 3, 463.

27 J. F. Quinn, A. P. R. Johnston, G. K. Such, A. N. Zelikin and F. Caruso, Chem. Soc. Rev., 2007, 36, 707.

28 J. Chen, C. Glaus, R. Laforest, Q. Zhang, M. Yang, M. Gidding, M. J. Welch and Y. Xia, Small, 2010, 6, 811.

29 M. R. Jones, R. J. Macfarlane, B. Lee, J. Zhang, K. L. Young, A. J. Senesi and C. A. Mirkin, Nat. Mater., 2010, 9, 913.

30 R. Jin, G. Wu, Z. Li, C. A. Mirkin and G. C. Schatz, J. Am. Chem. Soc., 2003, 125, 1643.

31 M. Rycenga, J. M. McLellan and Y. Xia, Adv. Mater., 2008, 20, 2416.

32 Y. G. Sun and Y. N. Xia, Science, 2002, 298, 2176.

33 M. Rycenga, C. M. Cobley, J. Zeng, W. Li, C. H. Moran, Q. Zhang, D. Qin and Y. Xia, Chem. Rev., 2011, 111, 3669.

34 B. J. Wiley, I. H. Sang, Z. Y. Li, J. McLellan, A. Siekkinen and Y. Xia, J. Phys. Chem. B, 2006, 110, 15666.

35 M. Lisunova, N. Holland, O. Shchepelina and V. V. Tsukruk, Langmuir, 2012, 28, 13345.

36 M. Lisunova, M. Mahmoud, N. Holland, Z. Combs, M. A. ElSayed and V. V. Tsukruk, J. Mater. Chem., 2012, 22, 16659.

37 R. Jin, G. Wu, Z. Li, C. A. Mirkin and G. C. Schatz, J. Am. Chem. Soc., 2003, 125, 1643.

38 B. T. Draine and P. J. Flatau, J. Opt. Soc. Am. A, 1994, 11, 1491. 39 M. Hu, J. Chen, Z. Y. Li, L. Au, G. V. Hartland, X. Li, M. Marqueze and Y. Xia, Chem. Soc. Rev., 2006, 35, 1084.

40 M. A. Mahmoud and M. A. El-Sayed, J. Phys. Chem. B, 2013, 117, 4468.

41 M. A. Mahmoud and M. A. El-Sayed, J. Phys. Chem. C, 2011, 115, 12726.

42 T. A. F. König, P. A. Ledin, J. Kerszulis, M. A. Mahmoud, M. A. El-Sayed, J. R. Reynolds and V. V. Tsukruk, ACS Nano, 2014, 8, 6182. 
43 P. Obreja, D. Cristea, E. Budianu, R. Gavrila, M. Kusko and V. Kuncser, IEEE Microelectronics, ICM 2004 Proceedings. The 16th International Conference, 2004.

44 S. Gomez-Grana, J. Perez-Juste, R. A. Alvarez-Puebla, A. Guerrero-Martinz and L. M. Liz-Marzan, Adv. Opt. Mater., 2013, 1, 477.

45 M. A. Mahmoud and M. A. El-Sayed, J. Am. Chem. Soc., 2010, 132, 12704.

46 M. A. Mahmoud and M. A. El-Sayed, Nano Lett., 2009, 9, 3025. 47 C. L. Nehl, H. W. Liao and J. H. Hafner, Nano Lett., 2006, 6, 683.

48 M. Lisunova, X. W. Wei, D. DeJarnette, G. T. Forcherio, K. R. Berry, P. Blake and D. K. Roper, RCS Adv., 2014, 4, 20894.

49 D. K. Roper, W. Ahn and M. Hoepfner, J. Phys. Chem. C, 2007, 111, 3636.

50 K. Berry, A. Russell, P. Blake and D. K. Roper, Nanotechnology, 2012, 23, 375703.

51 P. K. Jain, K. S. Lee, I. H. El-Sayed and M. A. El-Sayed, J. Phys. Chem. B, 2006, 110, 7238.

52 X. Chen, Y. Chen, M. Yan and M. Qiu, ACS Nano, 2012, 6, 2550.

53 A. O. Govorov, W. Zhang, T. Skeini, H. Richardson, J. Lee and N. A. Kotov, Nanoscale Res. Lett., 2006, 1, 84.

54 V. Kotaidis, C. Dahmen, G. von Plessen, F. Springer and A. Plech, J. Chem. Phys., 2006, 124, 184702.

55 A. G. Russell, M. McKnight, J. Hestekin and D. K. Roper, Langmuir, 2011, 27, 7799.

56 A. Russell, M. McKnight, A. Sharp, J. Hestekin and D. K. Roper, J. Phys. Chem. C, 2010, 114, 10132.

57 A. O. Govorov and H. Hugh, Nano Today, 2007, 2, 3.

58 A. O. Govorov, W. Zhang, T. Skeini, H. Richardson, J. Lee and N. A. Kotov, Nanoscale Res. Lett., 2006, 1, 84.

59 J. R. Dunklin, G. T. Forcherio, K. R. Berry and D. K. Roper, ACS Appl. Mater. Interfaces, 2013, 5, 8457.

60 L. R. Hirsch, R. J. Stafford, J. A. Bankson, S. R. Sershen, R. E. Price, J. D. Hazle, N. J. Halas and J. L. West, Proceedings of the Second Joint EMBS/BMES Conference, 2002.
61 X. Huang, P. K. Jain, I. H. El-Sayed and M. A. El-Sayed, Lasers. Med. Sci., 2008, 23, 217.

62 I. H. El-Sayed, X. Huang and M. A. El-Sayed, Cancer Lett., 2006, 239, 129.

63 X. Huang, P. K. Jain, I. H. El-Sayed and M. A. El-Sayed, Photochem. Photobiol., 2006, 82, 412.

64 X. Huang, P. I. H. El-Sayed and M. A. El-Sayed, J. Am. Chem. Soc., 2006, 128, 2115.

65 N. Grillet, D. Manchon, F. Bertorelle, C. Bonnet, M. Broyer, E. Cottancin, J. Lerme, M. Hillenkamp and M. Pellarin, ACS Nano, 2011, 5, 9450.

66 J. Chen, C. Glaus, R. Laforest, Q. Zhang, M. Yang, M. Gidding, M. J. Welch and Y. Xia, Small, 2010, 6, 811.

67 Y. Mamunya, A. Boudenne, N. Lebovka, L. Ibos, Y. Candau and M. Lisunova, Compos. Sci. Technol., 2008, 68, 1981.

68 V. Datsyuk, M. Lisunova, M. Kasimir, S. Trotsenko, K. Gharagozloo-Hubmann, I. Firkowska and S. Reich, Appl. Phys. A: Mater. Sci. Process., 2011, 105, 781.

69 V. Datsyuk, I. Firkowska, K. Gharagozloo-Hubmann, M. Lisunova, A.-M. Vogt, A. Boden, M. Kasimir, S. Trotsenko, G. Czempiel and S. Reich, 27th IEEE SEMITHERM Symposium, 2011, p. 325.

70 A. M. Mahmoud, D. O'Neil and M. A. El-Sayed, Chem. Mater., 2014, 26, 44.

71 Q. Zhang, W. Li, L.-P. Wen, J. Chen and Y. Xia, Chem.-Eur. J., 2010, 16, 10234.

72 J. Chen, M. Yang, Q. Zhang, E. C. Cho, C. M. Cobley, C. Kim, C. Glaus, L. V. Wang, M. Welch and Y. Xia, Adv. Funct. Mater., 2010, 20, 3684.

73 M. O. Lisunova, I. Drachuk, O. A. Shchepelina, K. D. Anderson and V. V. Tsukruk, Langmuir, 2011, 27, 11157.

74 R. Suntivich, O. Shchepelina, I. Choi and V. V. Tsukruk, ACS Appl. Mater. Interfaces, 2012, 4, 3102.

75 P. J. Flatau and B. T. Draine, Opt. Express, 2012, 20, 1247.

76 P. B. Johnson and R. W. Christy, Phys. Rev. B: Solid State, 1972, 57, 783. 